\title{
Panel Discussion
}

Mechanisms responsible for initiation and progression of coronary heart disease

\section{An Angioscopic Evaluation of the Pathogenesis of Ischemic Heart Disease and the Mechanisms of Coronary Artery Disease Progression*}

\author{
Kyoichi Mizuno**, Kimio Satomura**, Toshio Shibuya**, Ko AraKawa**, \\ Hirotsugu TABATA**, Hirokuni EtsudA**, Akira KurITA** and Haruo NaKamura**
}

\begin{abstract}
Although coronary arteriography is the method of choice for documenting the extent and degree of coronary arteriosclerotic lesions, it is unable to clearly detect intraluminal changes, such as a plaque rupture and thrombosis, that characterize an active lesion morphology associated with acute coronary disorders $^{1-5)}$. In contrast, however, coronary angioscopy is far more sensitive than arteriography in detection of intracoronary structures ${ }^{1,6)}$. Further, unlike a histopathologic examination, angioscopy also makes it possible to examine the time course changes in the coronary lumen?).

Thus, the purpose of this study is to investigate the pathogenesis of acute coronary disorders such as an unstable angina, an acute myocardial infarction, and a vasospastic angina by using coronary angioscopy. Also, the progression of coronary stenosis in patients with an unstable or stable angina has been studied.
\end{abstract}

\section{Patients and Methods}

\section{The pathogenesis of ischemic heart disease}

During cardiac catheterization, percutaneous transluminal coronary angioscopy was performed in 180 consecutive patients anatomically suitable for angioscopy and good pictures for analysis were obtained in 134 of those subjects. The cases analyzed consisted of 22 patients with an acute myocardial infarction (within 8 hours of onset), 28 with a recent myocardial infarction ( 2 days to 2 months since onset), 37 with an old myocardial infarction, 18 with an unstable angina, 23 with a stable angina, and 6 with a vasospastic angina.

Several factors were taken into consideration in determining the site of the angioscopy. In cases of a single vessel disease, the selected target lesion was

* This paper was presented at the 23rd annual meeting of the Japan Atherosclerosis Society in June 1991 in Kobe

** First Department of Internal Medicine, National Defense Medical College an ischemia-related artery and the most severe stenotic lesion was observed. In case of a multivessel disease, other criteria were used to determine the site of the angioscopy. In those with an acute myocardial infarction or an unstable angina, the infarctionrelated or ischemia-related artery was defined as being the occluded or significantly stenotic vessel that corresponded most closely to the ischemic area, as determined by ST-T segment changes during pain at rest. In those with a recent myocardial infarction or an old myocardial infarction, the infarction-related artery was determined by electrocardiography, a left ventricular wall motion abnormality and/or by thallium-201 myocardial scintigraphy. In those with stable angina, the most severe stenotic lesion in a major coronary branch, as determined by thallium201 myocardial scintigraphy, was chosen for the angioscopy. In those with a variant form angina pectoris, the lesion that demonstrated a spastic reaction on being provoked by an intracoronary administration of acetylcholine was selected for observation by angioscopy.

Prior to angioscopic observation, informed written consent was obtained in all cases. Informatively, we defined a thrombus as being a coalescent red and/or white mass adhering to the intima and protruding into the inner lumen; an intimal irregularity as plaque with a ragged or irregular surface; and a xanthomatous plaque as plaque with solid yellow coloration that was distinguishable from the other intima. Thus, we did not consider flat yellow streaks as being xanthomatous plaque.

Angioscopic pictures were reviewed by 2 doctors unaware of the clinical data and arteriographic findings. Finally, angioscopy was performed by means of a new angioscope that incorporates a balloon at the distal tip and an angulation mechanism that was developed at our institute, the details of which and the angioscopic procedure used having been previously described ${ }^{8)}$. 


\section{Progression of coronary stenosis}

1) Unstable angina cases

We studied the progression of stenosis in the atherosclerotic coronary lesions of 10 patients who had previously undergone arteriography and were later hospitalized for an episode of unstable angina pectoris, and compared them to the findings in 10matched, stable angina patients who also had undergone prior coronary arteriography. The unstable angina and stable angina patients were similar with regard to the following parameters: age (mean: 52 and 51 years old, respectively); sex (7 men in each group); the interval between studies (mean \pm SD: $24.8 \pm 25.2$ and $26.8 \pm 24.1$ months, respectively); the number of risk factors (mean $\pm \mathrm{SD}: 1.9 \pm 0.8$ and $1.8 \pm 0.9$, respectively); and the percentage of diameter stenosis on initial arteriography (mean \pm SD: $43.2 \pm 33.6 \%$ and $43.8 \pm 38.2 \%$, respectively). Informatively, patients with either a new $Q$ wave or a myocardial infarction during the interval between the first and second arteriography were excluded from this study.

Coronary arteriography was performed immediately after an intracoronary administration of nitroglycerin, and the degree of stenotic progression was evaluated in the lesion that showed the greatest progression. The stenosis was considered to have progressed if the stenosis seen in the second study showed an increase of more than one grade (AHA classification) in comparison with the first study.

2) Relationship between the progression of the coronary stenosis and the plasma lipid levels in the stable angina patients

In the 102 patients with stable angina pectoris who underwent coronary arteriography two times interval: 2-84 months), the lipid levels, which included measurement of the total cholesterol, triglyceride, HDL-cholesterol, Apo A-I, Apo A-II, Apo B, Apo C-II, Apo C-III, and Apo E, were ascertained at the time of their first and second catheterization, and the first and second coronary angiograms were compared. The criterion used to indicate stenotic progression was as same as for the previous study. Further, the relationship between the progression of the coronary stenosis and the plasma lipid levels in the stable angina patients was studied.

\section{The statistical analysis}

The chi-square statistic was used to test the uniformity of the incidence rate of the thrombus, the irregular surface, and the xanthomatous plaque, which were confirmed by angioscopy, in the acute myocardial infarction patients, and stable angina patients, respectively. Because the result of the test was found significant at the 0.05 level, further comparisons were made between any two groups.

Dichotomous parameters in the stenotic progression study were evaluated by using McNemar's test, and the independent and dependent Student's t test were used for continuous variables. A p value $<0.05$ was considered significant.

\section{Results}

\section{The pathogenesis of an ischemic heart disease}

The angioscopic findings in 134 patients are summarized in Table 1. The frequency of thrombi was significantly higher in patients with an acute myocardial infarction, a recent myocardial infarction, and an unstable angina than in those with an old myocardial infarction and a stable angina. Thrombi were observed in all 11 cases of patients with a postinfarction angina.

In contrast, thrombi were observed in only 4 of 17 patients without a postinfarction angina $(\mathrm{p}<$ 0.001 ). The frequency of an intimal irregular surface, including a plaque rupture, an ulceration, and an intimal flap was also significantly higher in patients with an acute myocardial infarction, a recent myocardial infarction, and an unstable angina than in those with an old myocardial infarction and a stable angina.

Yellow atheromas were observed more frequently in patients with an acute myocardial infarction, a recent myocardial infarction and an unstable angina than in those with an old myocardial infarction and a stable angina. Intimal damage was observed in 3 out of 6 patients $(50 \%)$ with a vasospastic angina, and white smooth atheromas were observed in most

Table 1 Angioscopic findings in 134 patients

\begin{tabular}{|c|c|c|c|c|c|c|c|}
\hline & $\mathrm{N}$ & $\begin{array}{c}\text { AMI } \\
22\end{array}$ & $\begin{array}{c}\text { RMI } \\
28\end{array}$ & $\begin{array}{c}\text { OMI } \\
37\end{array}$ & $\begin{array}{c}\text { UA } \\
18\end{array}$ & $\begin{array}{l}\text { SA } \\
23\end{array}$ & $\begin{array}{c}\text { VSA } \\
6\end{array}$ \\
\hline Thrombus & & $21(95 \%)$ & $15(53 \%)$ & $1(3 \%)$ & $16(89 \%)$ & $0 \quad(0 \%)$ & $1(17 \%)$ \\
\hline Irregular surface & & $11(50 \%)$ & $13(46 \%)$ & $3(3 \%)$ & $8(44 \%)$ & $2(9 \%)$ & $3(50 \%)$ \\
\hline Xanthomatous plaque & & $11(50 \%)$ & $13(46 \%)$ & $4(11 \%)$ & $9(50 \%)$ & $4(17 \%)$ & $1(17 \%)$ \\
\hline
\end{tabular}

AMI: acute myocardial infarction; RMI: recent myocardial infarction; OMI: old myocardial infarction; US: unstable angina; SA: stable angina; VSA: vasospastic angina 
cases of those with a stable angina and an old myocardial infarction.

\section{Progression of coronary stenosis}

1) Unstable angina cases

Stenotic progression occurred in 9 of 10 patients with an unstable angina but in 5 of 10 patients with a stable angina. The percentage of the diameter stenosis increased from $43.6 \%$ to $92.7 \%$ in those with an unstable angina, and from $43.2 \%$ to $66.9 \%$ in those with a stable angina, thus indicating a significant difference in the degree of stenotic progression between the 2 groups (Fig. 1).

2) Relationship between the progression of coronary stenosis and the plasma lipid levels in patients with a stable angina

Forty-four patients were revealed as showing a stenotic progression of their coronary artery disease and 56 patients did not.

At both the first and the second arteriography, the values of the total cholesterol, Apo B, Apo C-II, Apo E, and Apo B/A-I were significantly higher in the stenotic progression group than in the group

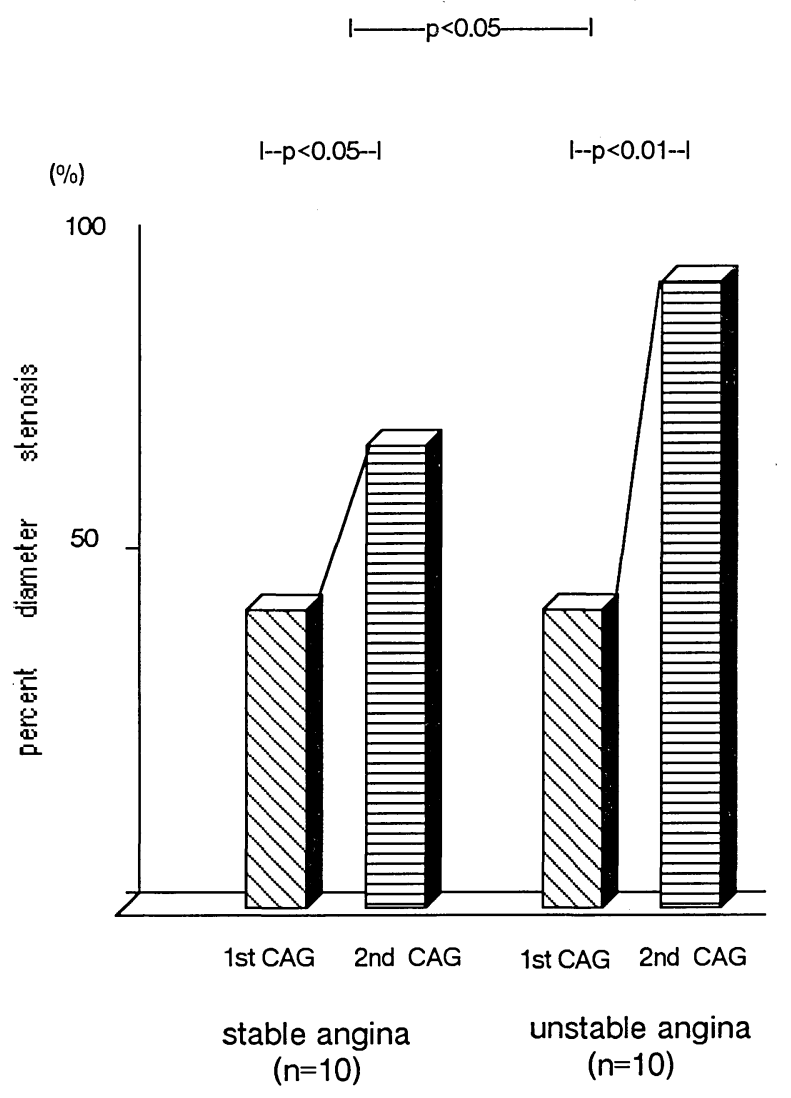

Fig. 1 Comparison of stenotic progression between unstable angina and stable angina in matched patients. showing no such progression. Figure 2 shows the effect of the total cholesterol on the progression of coronary stenosis. The value of the HDL cholesterol was significantly lower in the progression group than the non-progression group, whereas, at the first arteriography, the Apo C-III value was significantly higher in the progression group than in the nonprogression group.

\section{Discussion}

This angioscopic study confirms that thrombi overlying a rupture in the lining of the plaque may play a major role in an acute myocardial infarction, a postinfarction angina, and an unstable angina. It has been reported increased shear forces at the level of the stenosis, acute changes in coronary pressure related to exercise, and changes in the coronary tone may predispose plaque to disrupt ${ }^{2}$. In this regard, this study indicates that xanthomatous plaque, which is likely to be cholesterol rich with a fibrous cap that must be thin, may also be one of the important predispositions that leads to plaque rupture. It also has been found that exposed components of the arterial media, particularly fibrillar collagen, in

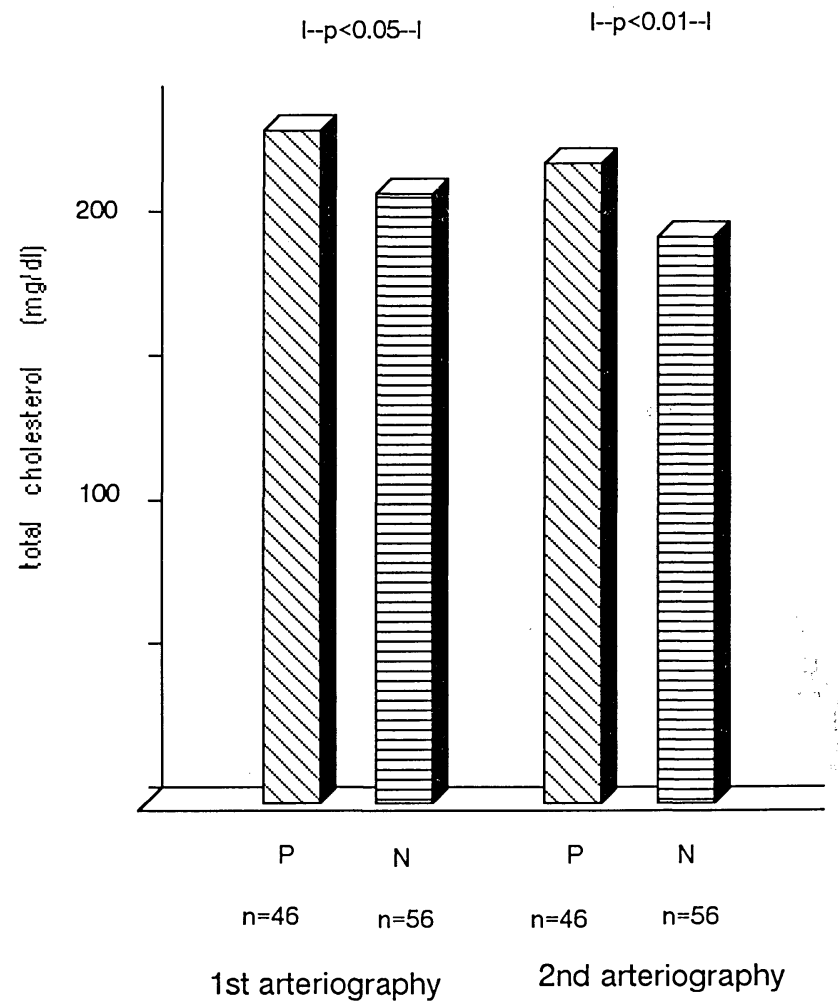

Fig. 2 Effect of total cholesterol on the progression of coronary stenosis.

$\mathbf{P}=$ progression, $\mathrm{N}=$ non progression 
addition to other mediators, induces platelet aggregation and coronary thrombosis ${ }^{2)}$. According to the another report, the major difference between an acute myocardial infarction and an unstable angina is the severity of the coronary obstruction ${ }^{9)}$. The thrombi in an unstable angina may be liable and not very large, and therefore seldom produce complete coronary obstruction.

After onset of an acute myocardial infarction, the intimal disruption heals rapidly. In this healing process, after the attachment of platelets, endothelial cells grow inwardly from the margins of the damaged surface and cover the platelet-fibrin mass. Simultaneously, the platelets and fibrin are rapidly replaced by macrophages, smooth muscle cells, and fibrous tissue, so that at 4 weeks the site of the prior intimal damage is often not readily identifiable ${ }^{9}$. However, a postinfarction angina occurred in patients who still have coronary thrombi. Healing of the intimal disruption leads to stabilization of the acute coronary syndrome.

This study also indicates that intimal damage plays one of the major roles in variant form angina pectoris. It has been found that in the disfunctional endothelium, vasoconstriction may occur by a reduction in the production of both an endotheliumderived relaxing factor (EDRF), due to histamine, serotonin, thrombin, and prostacyclin $\left(\mathrm{PGI}_{2}\right)^{10-13)}$. Vasoconstriction also may be provoked by the release of endothelium-independent vasoconstrictor substances from the platelets and thrombi $\mathrm{i}^{10-13)}$.

Additionally, this angiographic study demonstrates that an unstable angina is strongly associated with stenotic progression, depending on the extent and severity of the coronary obstruction. The progression of a coronary obstruction was much more frequently observed in the group with an unstable angina than in control patients with a stable angina who were matched for age, sex, and the extent of coronary artery disease. Our angioscopic study has revealed that an intimal irregularity and a thrombus were observed more frequently in patients with an unstable angina than in those with a stable angina. Therefore, the one of the causes of a stenotic progression in unstable angina patients must be a partially occlusive thrombosis that developed on the intimal irregularity. As has been reported, both thrombus incorporation and endothelial healing cause the rapid progression of coronary stenosis ${ }^{3}$.

It is well known that the serum lipid and lipoprotein levels are strongly and independently associated with the prevalence of a coronary artery disease ${ }^{14,15)}$. The level of apolipoprotein also is said to be a good discriminator between atherosclerotic subjects and controls $^{16)}$. In this regard, our study has shown that plasma lipid levels and apolipoproteins also may play an important role in the coronary artery stenotic progression in patients with a stable angina. Thus, a proper diet and drug therapy is thought necessary for patients with risk factors ${ }^{17)}$.

In conclusion, the presence of thrombi may be important factor in the pathogenesis of acute coronary disease and in the stenotic progression of a coronary obstruction in patients with an unstable angina. Further, the plasma lipid levels and the apolipoproteins appear to play an important role in the progression of a coronary artery stenosis in patients with a stable angina.

\section{References}

1) Mizuno, K., Miyamoto, A., Satomura, K., Kurita, A., Arai, T., Sakurada, M., Yanagida, S. and Nakamura, H.: Angioscopic coronary macromorphology in patients with acute coronary disorders. Lancet, 337: 809-812 (1991).

2) Fuster, V., Badimon, L., Cohen, M., Ambrose, J. A., Badimon, J. J. and Chesebro, J. H.: Insight into the pathogenesis of acute ischemic syndromes. Circulation, 77: 1213-1220 (1988).

3) Forrester, J. S., Litvack, F., Grundfest, W. and Hickey, A.: A perspective of coronary disease seen through the arteries of living man. Circulation, 75: 505-513 (1987).

4) Ambrose, J. A., Winters, S. L. and Arora, R. R.: Coronary angiographic morphology in myocardial infarction: A link between the pathogenesis of unstable angina and myocardial infarction. J. Am. Coll. Cardiol., 6: 1233-1238 (1985).

5) Davies, M. J. and Thomas, A.: Thrombosis and acute coronary artery lesions in sudden ischemic death. N. Engl. J. Med., 310: 1137-1140 (1984).

6) Sherman, C. T., Litvack, F., Grundfest, W. S., Lee, M., Hickey, A., Chaux, A., Kass, R., Blanche, C., Matloff, J., Morgenstern, L., Ganz, W., Swan, H.J.C. and Forrester, J.: Demonstration of thrombus and complex atheroma by in-vivo angioscopy in patients with unstable angina pectoris. N. Engl. J. Med., 315: 913-919 (1986).

7) Mizuno, K., Miyamoto, A., Isojima, K., Kurita, A., Senoo, A., Arai, T., Kikuchi, M. and Nakamura, H.: A serial observation of coronary thrombi in vivo by a new percutaneous transluminal coronary angioscope. Angiology, 42: 91-99 (1992).

8) Mizuno, K., Arai, T., Satomura, K., Shibuya, T., Arakawa, K., Okamoto, Y., Miyamoto, A., Kurita, A., Utsumi, A. and Takeuchi, K.: New percutaneous transluminal coronary angioscope. J. Am. Coll. Cardiol., 13: 363-368 (1989).

9) Mizuno, K., Satomura, K., Miyamoto, A., Arakawa, K., Shibuya, T., Arai, T., Kurita, A., Nakamura, $\mathbf{H}$. and Ambrose, J. A.: Angioscopic evaluation of the 
appearance of coronary thrombus in acute coronary syndromes. N. Engl. J. Med., 326: 282-291 (1992).

10) Shimokawa, H., Aarhus, L. L. and Vanhoutte, P. M.: Porcine coronary arteries with regenerated endothelium have a reduced endothelium-dependant responsiveness to aggregating platelets and serotonin. Circ. Res., 61: 256-270 (1987).

11) Lamping, K. G., Marcus, M. L. and Dole, W. P.: Removal of the endothelium potentiates canine large coronary artery constrictor responses to 5 hydroxytryptamine in vivo. Circ. Res., 57: 46-54 (1985).

12)- Vanhoutte, P. M. and Shimokawa, H.: Endotheliumderived relaxing factor and coronary vasospasm. Circulation, 80: 1-9 (1989).

13) $\mathrm{Ku}, \mathrm{D}$. D.: Coronary vascular reacitvity after acute myocardial ischemia. Science, 218: 576-578 (1982).
14) Wilson, P. W., Garrison, R. J., Castelli, W. P., Feinleib, M., McNamara, P. M. and Kannel, W. B.: Prevalence of coronary heart disease in the Framingham offspring study: Role of lipoprotein cholesterols. Am. J. Cardiol., 46: 649-654 (1980).

15) Reed, D. M., Strong, J. P., Resch, J. and Hayashi, T.: Serum lipids and lipoproteins as predictors of atherosclerosis. Arteriosclerosis, 9: 560-564 (1989).

16) Avogaro, P., Bon, G. B., Cazzolato, G. and Quinci, G. B.: Are apolipoproteins better discriminators than lipids for atherosclerosis? Lancet, 901-903 (1979).

17) Satomura, K., Mizuno, K. and Shibuya, T.: Prevention of progression of coronary atherosclerosis by drug and diet therapy. Jpn. J. Geriat., 27 : 438-441 (1990).

\section{Summary}

Percutaneous transluminal coronary angioscopy was performed in 180 consecutive patients anatomically suitable angioscopy to investigate the pathogenesis of ischemic heart disease. Good pictures for analysis were obtained in 134 patients (22 with an acute myocardial infarction, 28 with a recent myocardial infarction, 37 with an old myocardial infarction, 18 with an unstable angina, 23 with a stable angina, and 6 with a vasospastic angina). Thrombi were observed in most patients with an acute myocardial infarction, a postinfarction angina, and an unstable angina. Yellow atheromas were observed more frequently in patients with an acute myocardial infarction, an unstable angina, and a recent myocardial infarction. The frequency of an intimal damage was significantly higher in patients with an acute myocardial infarction, an unstable angina, a recent myocardial infarction, and a vasospastic angina.

The progression of stenosis in coronary lesions of 10 patients who had previously undergone arteriography and were later hospitalized for an episode of unstable angina, and compared them to the findings in 10-matched stable angina patients who had undergone prior coronary arteriography. Stenotic pro- gression occurred in 9 of 10 patients with an unstable angina but in 5 of 10 patients with a stable angina. And there was a significant difference in the degree of stenotic progression between 2 groups.

Relationships between the progression of the coronary stenosis and the plasma lipid levels in the stable angina were studied in 102 patients. Forty-four patients were revealed as showing a stenotic progression of their coronary artery disease and 56 patients did not. At both the first and the second arteriography, the values of the total cholesterol, Apo B, Apo C-II, Apo E and Apo B/A-I were significantly higher in the stenotic progression group than in the group showing no such progression.

In conclusion, the presence of thrombi may be important factor in the pathogenesis of acute coronary disease and in the stenotic progression of a coronary obstruction in patients with an unstable angina. Further, the plasma lipid levels and the apolipoproteins appear to play an important role in the progression of coronary artery stenosis in patients with a stable angina.

Key words: ischemic heart disease, angioscopy, stenotic progression, thrombus, plasma lipid level. 